\title{
A Simple and Accurate Magnetic Gradient Sensor Configuration Dedicated to Electrical Currents Measurements
}

\author{
A. Msaed*, 0. Chadebec, and J. Delamare \\ Grenoble Electrical Engineering Laboratory (G2Elab), Grenoble-INP, UJF, CNRS, \\ ENSe3-BP 46, 38402 Grenoble, France
}

\begin{abstract}
This paper deals with electrical current identification from stray magnetic field measurements. To satisfy industrial's needs, a new technology is emerging and can allow size and cost reductions of sensors. It consists in placing non-contact magnetic field sensors closes to conductors and deducing the currents' magnitude by inverting the Biot-Savart law. The theoretical study executed in this paper illustrates the advantage of the gradient measurements compared to the field measurement, especially for the rejection of homogeneous magnetic disturbance. It is showing also that the approach of magnetic gradient measurements greatly reduces non homogeneous magnetic disturbance. But, when implementing the practical application for the gradient measurement approach, many difficulties will be appearing; we will list it in the paper sections. Next, we will suppose a configuration solution able to subdue these difficulties; it is based on "round-trip" conductors and consists to laying the two magnetic sensors collinearly along the same axis above the conductor sections to apply gradient measurements. Afterwards, we will apply this configuration for a three-phase system by describing the matrix form, using known geometries and locations of magnetic sensors, and we will explain the manner in which to calculate the reconstruction of the currents which is done by solving the inverse problem. The "round-trip" configuration combined with a simple magnetic shielding and integrated technology can lead to the design of highly accurate and low-cost current sensors.
\end{abstract}

Keywords: Magnetic Sensor, Magnetic Gradient Measurements, Identification of Electrical Currents, Reducing of Disturbance Magnetic Field.

\section{INTRODUCTION}

With the increase of the demand for power monitoring, current sensors must be more and more compact, accurate, low-cost and with wide measurement ranges. Furthermore, various principles of current measurements have been studied. The measurement of the current with a shunt introduces many problems such as Joule losses, noise and non-isolated measurements. Current Transformers (CT) have been widely used, ${ }^{1}$ but a small (CT) has a problems with direct current measurements and a problems of consumption due to the feedback. The current sensor based on the Hall Effect presents a large linearity error. Other principles were also proposed in Refs. [2, 3], they are based on the detection of the vibration of the cable carrying the current to be identified or by the measurement of its temperature. In this paper, we are interested in the measurement of the current from point measurements of the magnetic fields.

Nevertheless, there are several difficulties with this method; Industrial environments are often situated within disturbing magnetic fields which can induce large measurement inaccuracies. At present, in a current sensor based on the measurement of magnetic fields, the major problem is to make sure that the measured field derives exclusively from the current to be identified. A single magnetic sensor is insufficient to reconstruct the current flowing in a conductor, due to the presence of the earth's magnetic field. Thus, the main problem resides in the number of magnetic sensors and the manner of their positioning around the conductors. In addition, system sensibilities to external magnetic perturbations lead to an increase in reconstructing errors especially when we have a conductor with a high current value nearby the current 
to be reconstructed. One solution is to shield the probes with ferromagnetic material. However, the complication size and cost of the sensors increases, ${ }^{4}$ particularly when using double shields. Finally, the limitations of the magnetic sensors affect the measurements, such as saturation of the sensors and misalignment when using directional magnetic sensors.

We will show the results of the theoretical study for the approach of field and gradient measurements for a simple conductor by taking into count the advantages and the disadvantages for each approach. The approach of magnetic gradient measurements greatly reduces magnetic disturbance. It consists in placing non-contact magnetic field sensors nearby the conductors and deducing the currents by inverting the Biot-Savart law. ${ }^{5}$ We will apply theses approaches for a three-phase system by illustrating the numerical results for the reconstruction of a small current in the presence of a homogeneous and non homogeneous magnetic field. But, when implementing the practical application for the gradient measurement approach, many difficulties will be appearing; we will list it in the following sections. Then, we will suppose a configuration solution able to subdue these difficulties; it is based on "roundtrip" conductors and consists to laying the two magnetic sensors collinearly along the same axis above the conductor sections to apply gradient measurements. Next, we will apply this configuration for a three-phase system by describing the matrix form and the manner in which to calculate the reconstruction of the currents.

\section{BASIC THEORY}

Let $I_{c}$ be a current flowing in an infinitely long conductor, a scalar magnetic sensor placed closed to the conductor measures the main tangential component of the magnetic field. The directional field sensor is placed at a distance of a few millimeters to the conductor $I_{c}$; it is represented by an arrow in (Fig. 1). Thanks to the 2D Biot-Savart law, we can easily compute the magnetic field due to $I_{c}$ :

$$
H=\frac{I_{c}}{2 \pi r}=k \cdot I_{c}
$$

Where $r$ is the distance between the conductor and the field sensor. By inverting the previous relation, it is possible to get the current value from the measurement of magnetic field $\left(H_{\text {meas }}\right)$ :

$$
I_{c}=k^{-1} \cdot H_{\text {meas }}
$$

However, in real applications, it is usual that the magnetic sensor will be exposed to external fields of unknown origin such as a nearby conductor carrying a high current. Let us consider an infinitely long disruptive conductor in which flows a high current $I_{d}\left(I_{d}=300 \cdot I_{c}\right)$ generating an additional field:

$$
H_{\text {meas }}=H_{I_{c}}+H_{I_{d}}
$$

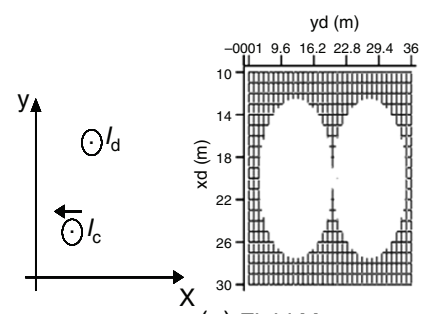

(a) Field Measurements

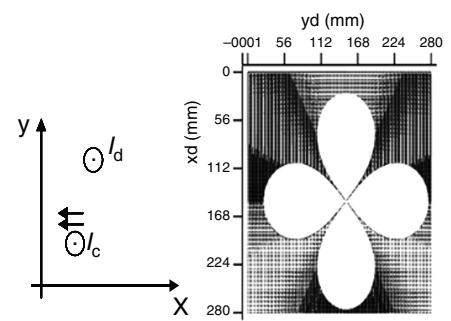

(b) Gradient Measurements

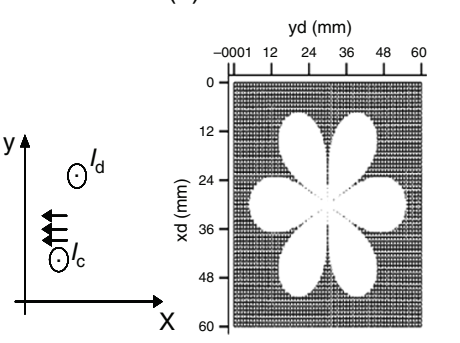

(c) Gradient of gradient Measurements

Fig. 1. Exclusion areas for the disturbing conductor with $\gamma<0.02$ for the three approaches. The field sensors, represented by an arrow, are positioned near to the conductor with the current to be measured $I_{c} ; I_{d}$ is the hard current value in the disruptive conductor.

We consider that the influence of this disruptive conductor is negligible if the ratio between the fields created by $I_{d}$ and $I_{c}$ is inferior to $2 \%$.

$$
\gamma=\frac{H_{I_{d}}}{H_{I_{c}}}<2 \%
$$

Then we can plot an exclusive area where the disruptive conductor should not be (Fig. 1(a)). This figure illustrates that $I_{d}$ must be placed at a distance higher than $16 \mathrm{~m}$ around the conductor $I_{c}$; an enormous distance. This condition is impractical to enforce in real industrial applications.

The approach of gradient measurements is based on the derivative of the magnetic field. In $2 \mathrm{D}$, the field generated by the conductor $I_{c}$ decrease as $1 / r^{2}$ for the gradient; compared to $1 / r$ dependence for field measurements. Furthermore, the gradient measurement naturally rejects constant external magnetic perturbations.

$$
\frac{d H}{d r}=\frac{-I_{c}}{2 \pi r^{2}}=M \cdot I_{c}
$$

In practice, this approach can be achieved by using two magnetic scalar sensors placed closed to each others (Fig. 1(b)) and by subtracting their two measured fields. 
In this computation, the gradient measurement is considered as being punctual. If we consider the previous case, where the influence of the disruptive conductor is negligible for $\gamma<0.02$, the exclusion area for disturbance conductor therefore decreases to $13 \mathrm{~cm}$. Notice that this gradient-based approach can be extended to the measurements of gradients of gradients by applying the second spatial derivative to the magnetic field:

$$
\frac{d^{2} H}{d r^{2}}=\frac{2 I_{c}}{2 \pi r^{3}}=L \cdot I_{c}
$$

Then, the field generated by $I_{c}$ has a $1 / r^{3}$ decrease law for gradient of gradients. It can be put into practice by using three field detectors placed close to each other as shown (Fig. 1(c)). For the above case, the exclusion area radius decreases to $3 \mathrm{~cm}$ (Fig. 1(c)).

\section{PHASES GENERALIZATION}

Considering three-phases, we have applied these three approaches for reconstructing a small current $I_{c}$ by supporting to the Biot-Savart law; $n$ magnetic sensors surround the system according to each approach. Thanks to the linearity of Biot-Savart law, each sensor will measure the sum of the magnetic field generated by the three currents.

$$
\begin{aligned}
& H_{1}=k_{11} \cdot I_{1}+k_{12} \cdot I_{2}+k_{13} \cdot I_{3} \\
& H_{2}=k_{21} \cdot I_{1}+k_{22} \cdot I_{2}+k_{23} \cdot I_{3} \\
& H_{n}=k_{n 1} \cdot I_{1}+k_{n 2} \cdot I_{2}+k_{n 3} \cdot I_{3}
\end{aligned}
$$

Then, we can write matrix system:

$$
\left[H_{i}\right]=\left[k_{i j}\right] \cdot\left[I_{j}\right] \quad(i=1 \ldots n)(j=1 \ldots 3)
$$

The coefficients of this matrix depend on the geometry of the conductors and the number of the field sensors and the locations. Once the geometry of the system is fixed, we can compute the matrix elements. This is done simply by imposing a known current through each conductor individually and computing the magnitude of the magnetic field from each field sensor. ${ }^{6}$ This method can be applied numerically if the geometry is more complex (by using Finite Element approach, for example), by in any case, the problem should be linear. Having determined the matrix coefficient the inverse problem is solved by the least squares approach. The system to solve becomes:

$$
\left(K^{T} K\right) \cdot I=K^{T} \cdot H
$$

Extracting the currents is accomplished by solving it.

The approach of field measurements is applied by using one magnetic field sensor for each conductor, whereas the gradient measurements is made by using two conductors placed closed to each other upon each conductor and by subtracting their measured fields. Then, to apply the
Table I. The relative error for the reconstructing of a small current $I_{c}$ in the presence of a homogeneous and non homogeneous magnetic field.

\begin{tabular}{lcc}
\hline & \multicolumn{2}{c}{ Relative error $(\%)$} \\
\cline { 2 - 3 } & $\begin{array}{c}\text { In the presence of } \\
\text { earth magnetic } \\
\text { field }(50 \mu \mathrm{T})\end{array}$ & $\begin{array}{c}\text { In the presence of a } \\
\text { disruptive conductor } \\
\left(I_{d}=300 \cdot I_{c}\right)\end{array}$ \\
\hline $\begin{array}{c}\text { Inversion field } \\
\text { measurements }\end{array}$ & 245 & 1016 \\
$\begin{array}{c}\text { Gradient measurements } \\
\text { Gradient of gradient } \\
\text { measurements }\end{array}$ & 0 & 55.6 \\
\hline
\end{tabular}

approach of gradient of gradient measurements for threephase sensors, we need three magnetic field sensors placed nearby the conductors, and the approach is executed by doing a double difference of the matrix element of field measurements. Besides, the distance which separates the magnetic sensors to the conductors is about a few millimeters in all these approaches. Two cases are studied, the first consists to reconstruct a small current $I_{c}$ in the presence of the earth's magnetic field $(50 \mu \mathrm{T})$, and in the second case, we add a disruptive conductor carrying a hard current $I_{d}\left(I_{d}=300 \cdot I_{c}\right)$ at a distance of $3 \mathrm{~cm}$ to the system, the relative errors incurred for the reconstruction of the small current $I_{c}$ basing to the three preceding approaches are given in Table I.

The approach of field measurement leads to an inexpensive current sensor but it presents a poor magnetic background rejection. Whereas, by applying the field gradient measurement, the relative error obtained for the reconstruction of a small current is zero percent in the presence of the earth's magnetic field. This result is obvious because uniform magnetic disturbances are naturally subtracted. Then, we notice a better background rejection in the presence of the disruptive conductor $I_{d}\left(I_{d}=300 \cdot I_{c}\right)$. One the side to these advantages, the gradient measurement solution is more expensive, and we will show in the following section, that the position of the directive magnetic sensor is very complex and it will affect on the computation of the relative error. However, the gradient of gradient measurements method improves significantly the rejection of an external magnetic disturbance, but the cost of the sensor therefore increases with using nine magnetic sensors and we note that the positioning of magnetic sensor is also critical. In the section three, we will detail the influence of misalignment of directive magnetic sensors on the computation of the relative error.

\section{DIFFICULTIES OF IMPLEMENTING THE PRACTICAL APPLICATION FOR THE GRADIENT MEASUREMENT}

When implementing the practical application for the approach of gradient measurements, many difficulties appear: the magnetic gradient should be measured along 


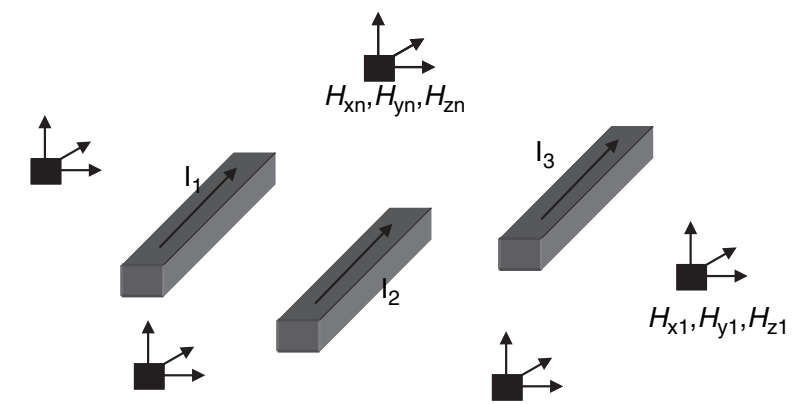

Fig. 2. $n$ magnetic field sensors surrounding three conductors.

one single collinear axis; any offset introduces by the magnetic sensors will affect the computation of relative error and the rejection of disturbance fields is thus inadequate. By positioning a directive magnetic sensor at a distance $d$ to the conductor crossing by the current to be reconstructed $I_{c}$, to apply the approach of gradient measurement, we placed another directive magnetic sensor nearby the first magnetic sensor. The distance which separates the two magnetic sensors is $\varepsilon$. In practical application, an offset can be executed. If we suppose here, that the second magnetic sensor presents an offset $\theta$ in comparison to the initial setting (Fig. 3). The relative error due to this offset is not negligible as illustrate (Fig. 4). The relative error incurred for an offset of $1^{\circ}$ is $2.3 \%$ in a cleaning magnetically environment and $2.74 \%$ in the presence of the disruptive conductor $\left(I_{d}=300 \cdot I_{c}\right)$. It's higher than the relative error required $2 \%$. However, the subtracting of the two field measurements in 2D is given by (10):

$$
\Delta B_{x}=\frac{\mu_{0} I_{c}}{2 \pi} \cdot \frac{-\varepsilon}{d(\varepsilon+d)}
$$

If $c$ is the measured error committed on the subtracting magnetic field, the relative error for the reconstructing of $I_{c}$ is:

$$
e=c \cdot d \cdot\left(1+\frac{d}{\varepsilon}\right)
$$

As a conclusion, a thin distance between the magnetic field sensors will increase the percentage of the relative

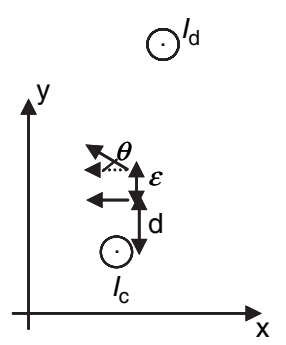

Fig. 3. A misalignment of one directive magnetic sensor is executed (two magnetic field sensors are represented by two arrows, in dotted we present the initial position of the directive magnetic sensor; $d$ is the distance which separate the first magnetic sensor to the conductor to be measured $I_{c}$, and $\varepsilon$ is the distance between the two magnetic sensors).

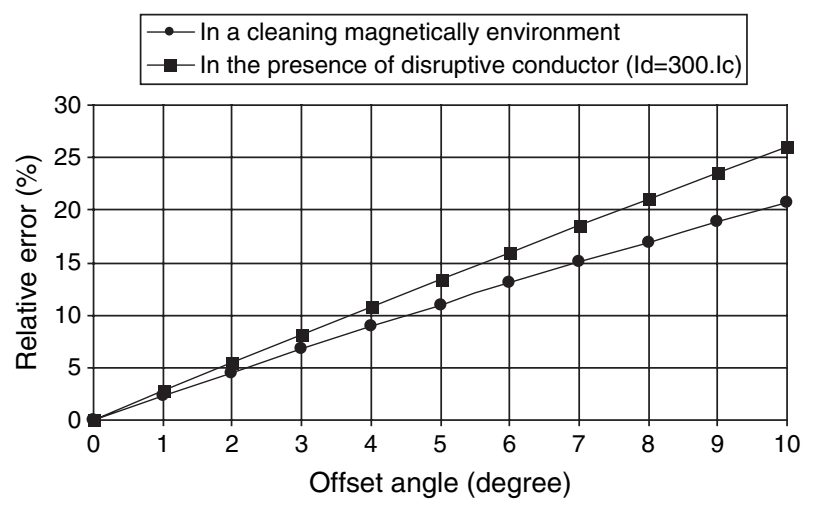

Fig. 4. The relative error due to the misalignment of one magnetic field sensor.

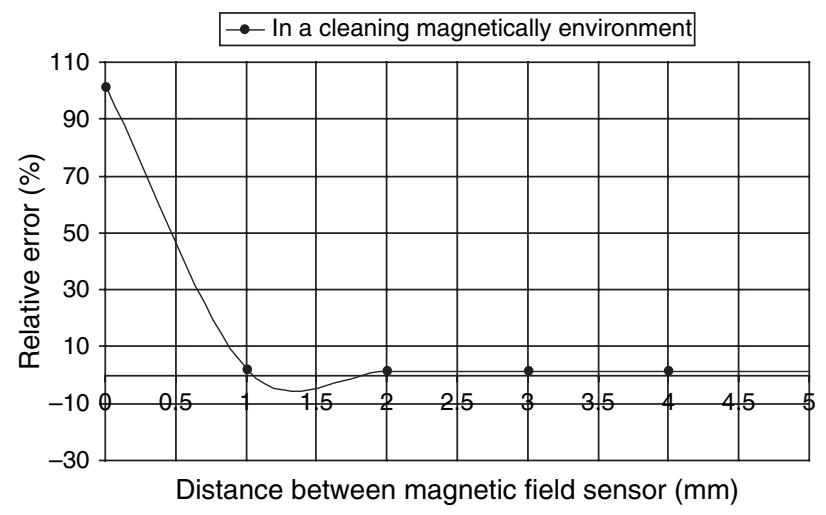

Fig. 5. The relative errors function on the distance which separate the magnetic field sensors.

error taking into count the measured error. Then, if we increase the distance which separates the magnetic field sensors, the approach of gradient measurement is not thus reach its aim; because to assure a good rejection of magnetic disturbance, the two magnetic field sensors should be placed closed to each other.

The Figure 5 shown the relative error incurred when the measured error $c$ is equal to $1 \%$ and in a cleaning magnetically environment; more the distance between the magnetic sensors is higher the relative error decrease but it is lead to a poor magnetic background rejection. In the following sections, we will search a solution to realize the practical application for the approach of gradient measurement, able to subdue the disadvantages of the above sensor/conductors configuration and having a satisfactory cost.

\section{IMPLEMENTATION OF GRADIENT MEASUREMENT WITH "ROUND-TRIP" CONDUCTORS}

The approach of gradient measurements can be also applied by using other configurations. If we suppose, that 


$$
\text { (a) }
$$
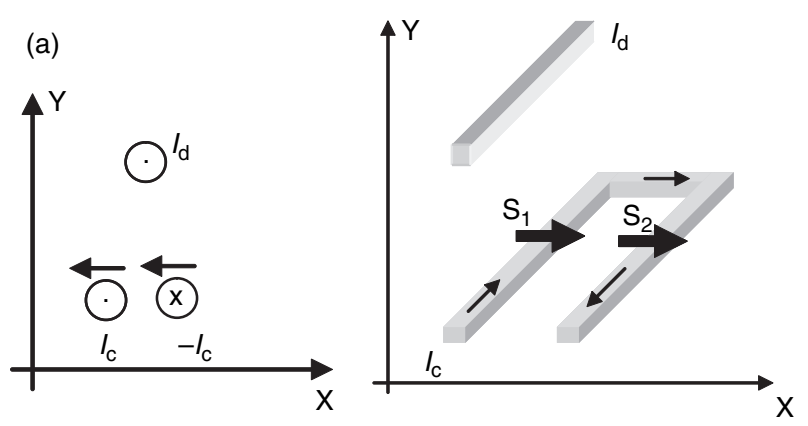

yd (mm)

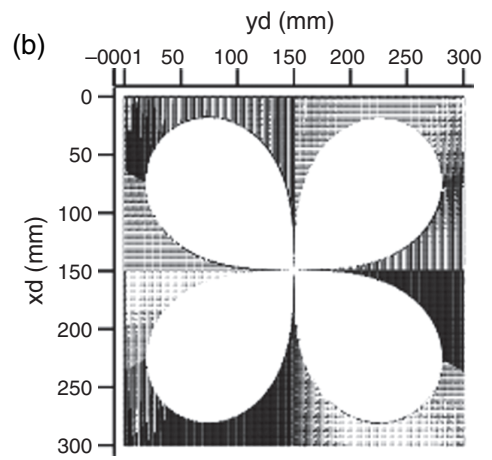

Fig. 6. (a) Two magnetic field sensors represented by two arrows are positioned above the sections of "round-trip" conductor flowing by the current to be measured $I_{c} ; I_{d}$ is the disruptive conductor carrying a hard current value. (b) Exclusion areas for disturbance conductor with $\gamma<$ 0.02 for measurements.

the conductor is made in a round-trip configuration, and by positioning one field sensor above each conductor section (Fig. 6(a)), we can reconstruct the current flowing through the conductor by subtracting the two measured fields. The theoretical study for this configuration predicts the same results for rejection of magnetic disturbances. To get a relative error of $2 \%$ for reconstruction $I_{c}$, the disruptive conductor $I_{d}\left(I_{d}=300 \cdot I_{c}\right)$ should be placed at a distance higher than $13 \mathrm{~cm}$. Figure 7 illustrates the model using six field detectors for three "round-trip" conductors to apply the gradient measurement method.

The distance which separates each conductor sections is also a few millimeters. Once the geometry of the system is fixed, we can express the matrix for the approach of magnetic gradient measurement by subtracting two magnetic inductions for two field detectors collinearly along the same axis above the conductor. The new matrix form is given in (12).

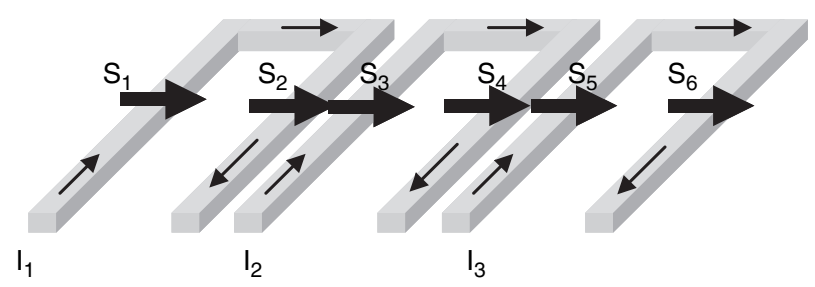

Fig. 7. Three-phase sensors with configuration "round-trip".

$$
\left[\begin{array}{l}
H_{1}-H_{2} \\
H_{3}-H_{4} \\
H_{5}-H_{6}
\end{array}\right]=\left[\begin{array}{lll}
M_{11} & M_{12} & M_{13} \\
M_{21} & M_{22} & M_{23} \\
M_{31} & M_{32} & M_{33}
\end{array}\right] \cdot\left[\begin{array}{c}
I_{1} \\
I_{2} \\
I_{3}
\end{array}\right]
$$

Where $M_{i j}$ is the difference of two elements $k_{i j}$ in the matrix of field measurements. These coefficients $k_{i j}$ depend on the geometry of the conductors, the distance between the conductor and the sensors and the distance between the conductor sections.

In the presence of the earth's magnetic field, the relative error incurred for the reconstruction of a small currant is zero percent. This result assures the interest of the gradient measurement to reject the uniform magnetic disturbance. Otherwise, if we placed at $3 \mathrm{~cm}$ a disruptive conductor carrying a hard current value $I_{d}\left(I_{d}=300 \cdot I_{c}\right)$, the relative error for $I_{c}$ decreases to $27 \%$ (From $1016 \%$ field measurement). The results show an improvement of the rejection of non-uniform magnetic disturbance.

This configuration subdues the problem of misalignment of magnetic sensor, because their positioning is very easy mechanically because each couple of magnetic field sensor will be mounted on the same layout. Then, we suppose that $c$ is the measured error committed on the subtracting magnetic field given by the relation (13) where $p$ is the distance between the conductor sections and $d$ the distance that separate the magnetic sensors from the conductor sections.

$$
\Delta B x=\frac{\mu_{0} I_{c}}{2 \pi} \cdot \frac{-2 p^{2}}{d\left(p^{2}+d^{2}\right)}
$$

The relative error for the reconstruction of $I_{c}$ is more lower than the previous case, where the two magnetic sensors were placed closed to each other, and it is given by (14):

$$
e=c \cdot d \cdot\left(\frac{1}{2}+\frac{d^{2}}{2 p^{2}}\right)
$$

As we conclude, a thin distance between the conductor sections will increase the percentage of the relative error taking into count the measured error, but with this configuration, the relative error is multiply by a factor $1 / 2$ instead a factor 1 as in the preceding case. Then, this relative error will greatly decrease. Besides, if we increase the distance which separates the conductor sections, we will then increase the dimension of the current sensors what is conflicting with size reducing aim of this new technology. The Figure 8 shown the relative error incurred when the measured error $c$ is equal to $1 \%$ and in a cleaning magnetically environment; more the distance between the conductor sections is higher the relative error decrease but the dimension of the current sensor rise against the preceding case.

We can conclude that the application of the approach of gradient measurement with "round-trip" configuration 


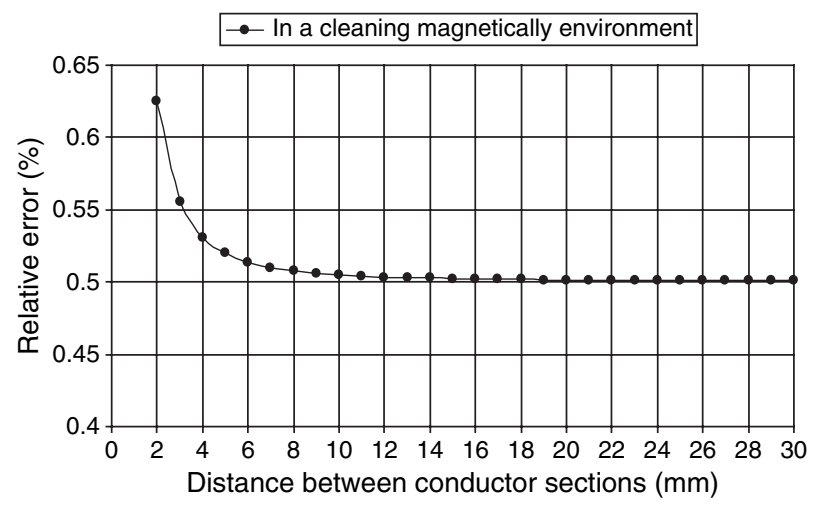

Fig. 8. The relative errors function on the distance which separate the conductor sections.

presents a good magnetic background rejection and subdue the disadvantages of the above sensor/conductors configuration having two magnetic sensors placed closed to each other nearby the conductor crossing by the current to be measured. Then, with round-trip conductor we can use a directive magnetic sensor without a problem of misalignment because it is placed on the same layout. Finally, with a distance of a few millimeters between the conductor sections, the relative error incurred is thus satisfactory. However, the addition of a simple magnetic shielding to the configuration "round-trip" can lead to the design of highly accurate and low cost current sensors, and in this case the material of shielding isn't saturate.

\section{CONCLUSION}

A method for non-contact current measurement based on magnetic gradient measurement is described. Several field probes in close proximity to a current carrying conductor are combined to measure the spatial derivative of the magnetic field, and which leads to a more accurate evaluation of the current. (We have demonstrated the advantages of this approach by the theoretical study for a one conductor case.) Furthermore, we have extended the methodology for the case of three phases; the numerical results have shown a much improved rejection of the homogeneous perturbations with the use of gradient measurements. In addition, in the presence of a disruptive conductor placed at $3 \mathrm{~cm}$ and carrying a hard current value $\left(I_{d}=300 \cdot I_{c}\right)$, we have reconstructed a small current $I_{c}$ with a lower relative error by applying the gradient approach. However, when implementing the practical application for the gradient measurement approach, many difficulties will be appearing as the misalignment of directive magnetic sensors and the influence of the distance between their on the computation of the relative error for reconstructing the currents. Next, we have proposed a solution able to subdue the upper difficulties; it is based on "round-trip" conductors and consists to laying the two magnetic sensors collinearly along the same axis above the conductor sections to apply gradient measurements. This configuration was showing a good magnetic background rejection and a satisfactory cost. Besides, with round-trip conductor we can use a directive magnetic sensor without a problem of misalignment because it is placed on the same layout. Finally, the addition of a simple magnetic shielding to the configuration "round-trip" can lead to the design of highly accurate and low cost current sensors.

Acknowledgments: The authors would like to thank "La METRO de Grenoble" who supported this work.

\section{References and Notes}

1. V. Frick, L. Hebrard, P. Poure, F. Anstotz, and F. Braun, IEEE Sens. J. 3 (2003).

2. A. V. Mamishev, S. X. Short, T. W. Kao, and B. D. Russell, IEEE Trans. On Instrumentation and Measurement 45, 457 (1996).

3. J. R. Kinard, T. E. Lipe, C. B. Childers, D. B. Novontny, and D. X. Huang, IEEE Trans. On Instrumentation and Measurement 46, 391 (1997).

4. T. Kudo, N. Tsuji, T. Asada, S. Sugiyama, and S. Wakui, IEEE Trans. on Magnetics 42, 3362 (2006).

5. G. D'Antona, L. Di Rienzo, R. Ottoboni, and A. Manara, IEEE Trans. on Instrumentation and Measurements 50, 1289 (2001).

6. E. R. Olson and R. D. Lorenz, Industry Application Conferences (2007), pp. 1426-1433. 